\title{
Identification of host-microbe interaction factors in the genomes of soft rot-associated pathogens Dickeya dadantii 3937 and Pectobacterium carotovorum WPP14 with supervised machine learning
}

\author{
Bing $\mathrm{Ma}^{1,4^{*}}$, Amy O Charkowski ${ }^{2}$, Jeremy D Glasner ${ }^{1}$ and Nicole T Perna ${ }^{1,3}$
}

\begin{abstract}
Background: A wealth of genome sequences has provided thousands of genes of unknown function, but identification of functions for the large numbers of hypothetical genes in phytopathogens remains a challenge that impacts all research on plant-microbe interactions. Decades of research on the molecular basis of pathogenesis focused on a limited number of factors associated with long-known host-microbe interaction systems, providing limited direction into this challenge. Computational approaches to identify virulence genes often rely on two strategies: searching for sequence similarity to known host-microbe interaction factors from other organisms, and identifying islands of genes that discriminate between pathogens of one type and closely related non-pathogens or pathogens of a different type. The former is limited to known genes, excluding vast collections of genes of unknown function found in every genome. The latter lacks specificity, since many genes in genomic islands have little to do with host-interaction.
\end{abstract}

Result: In this study, we developed a supervised machine learning approach that was designed to recognize patterns from large and disparate data types, in order to identify candidate host-microbe interaction factors. The soft rot Enterobacteriaceae strains Dickeya dadantii 3937 and Pectobacterium carotovorum WPP14 were used for development of this tool, because these pathogens are important on multiple high value crops in agriculture worldwide and more genomic and functional data is available for the Enterobacteriaceae than any other microbial family. Our approach achieved greater than $90 \%$ precision and a recall rate over $80 \%$ in 10 -fold cross validation tests.

Conclusion: Application of the learning scheme to the complete genome of these two organisms generated a list of roughly 200 candidates, many of which were previously not implicated in plant-microbe interaction and many of which are of completely unknown function. These lists provide new targets for experimental validation and further characterization, and our approach presents a promising pattern-learning scheme that can be generalized to create a resource to study host-microbe interactions in other bacterial phytopathogens.

Keyword: Pattern recognition, Data mining, Plant pathogen, Genome scale analysis, Enterobacteria

\footnotetext{
*Correspondence: bma@som.umaryland.edu

'Genome Center of Wisconsin, University of Wisconsin-Madison, Madison, WI 53706, USA

${ }^{4}$ Current affiliation: Institute for Genome Sciences, University of Maryland

School of Medicine, Baltimore, MD 21201, USA

Full list of author information is available at the end of the article
} 


\section{Background}

Interactions between plant-associated microbes and their eukaryotic hosts are complex biological processes involving hundreds, if not thousands, of genes from each organism. Understanding the molecular mechanisms of such complex processes at the systems-scale is seriously hampered by the lack of a comprehensive list of gene products that contribute for even a single bacterial or fungal pathogen. Variation in lifestyles and pathogenic potential between organisms makes the challenge all the greater. Genome sequencing has dramatically increased the potential for large-scale screens to identify genes involved with host-microbe interactions. Direct experimental evidence is the obvious gold standard, but not all significant pathogens are experimentally tractable, and selection of experimental conditions and convenient hosts for high-throughput screens can limit discovery. More targeted experiments can be designed to probe function more completely, but these are time consuming and generally limited to a smaller number of candidate genes. Further, it is unclear what experiments to conduct if a candidate gene is of completely unknown function. Importantly, genes of unknown function make up a substantial fraction of each sequenced genome, and it is likely that among these lie some of the greatest potential for discovery of truly novel aspects of host-microbe interaction (as well as many other complex biological processes).

Computational approaches to identify potential hostmicrobe interaction factors and predict their specific functions can be a valuable way to guide experimentation, and may be the only option for some recalcitrant organisms. Typical bioinformatics strategies include searching for sequence similarity to gene products known to contribute to host-microbe interaction in other organisms, and comparing genomes to identify gene islands that discriminate between pathogens of one type and closely related nonpathogens or pathogens of a different type. Both strategies are useful, but the former is limited to known genes and detectable levels of sequence similarity, and thus excludes the vast collections of genes of unknown function. The latter lacks specificity, since many genes in genomic islands may have little to do with host interactions, and the definition of rules for the distribution across organisms can be arbitrary. There are no simple rules to define the relevant distribution for the set of orthologous genes across genomes, especially when there are a large number of genomes being compared. Further, it is preferable in many situations to factor in other features such as genome context or gene expression data as additional evidence sources to predict whether a gene is associated with host-microbe interaction processes.

More sophisticated computational prediction strategies can introduce a variety of other types of evidence, but integration of diverse data types remains a challenge. Machine learning techniques are ideally suited for pattern recognition tasks to accommodate diverse biological data sources into a single predictive analysis to achieve superior performance over any individual type of data, especially where (1) data sets are large, (2) with heterogeneous sources, and (3) patterns are not easily described by a compact set of rules, all of which are true for the task of genome-scale identification of host-microbe interaction factors. Supervised machine learning schemes have been receiving increasing attention recently as a promising approach to study diverse biomedical problems [1-5], but no previous study focused on host-microbe interaction factors. In this study, we developed a supervised machine learning strategy to identify the gene inventory involved with host-microbe interaction from two soft rot-associated enterobacteria, Dickeya dadantii (aka. Erwinia chrysanthemi) 3937 [6], and Pectobacterium carotovorum (aka. Erwinia carotovorum) WPP14 [7]. Our approach allows us to incorporate a wide variety of input data, including homology information, genome context, predicted transcription factor binding sites, and microarray transcript profiles. It has achieved promising results with precision rate over $90 \%$ with recall rate over $80 \%$. Further, our study generates an extended list of roughly 200 candidate interaction factors and provides experimentally testable hypotheses to stimulate further research on the molecular mechanisms of soft rot pathogenesis and survival in plant hosts. This study represents a promising application of pattern-recognition methods for identification of factors involved in complex biological processes, which can be generalized to study other plant-associated organisms.

\section{Methods}

\section{Target genome selection}

Soft rot-associated enterobacteria are economically important pathogens that infect a broad range of plant species [8-11]. Soft rot bacterial pathogenesis is characterized by rapid necrosis of parenchymatous tissues, mainly due to the action of secreted enzymes that degrade the middle lamellae and the primary cell wall [12]. Continuing discovery of additional genes involved in survival in a plant host or which contribute directly to pathogenesis [13-19] suggests that even for well-studied organism such as Dd3937, we have not yet achieved a comprehensive list of hostmicrobe interaction factors or a complete understanding of their precise roles. In this study, we target two soft rotassociated phytopathogens for genome-wide identification of host interaction factors (Table 1). One, Dickeya dadantii 3937 (Dd3937) was originally isolated from Saintpaulia ionantha $[20,21]$, and is a long-standing model system for this group of organisms [6]; the other, Pectobacterium carotovorum carotovorum WPP14 was isolated from infected potato in Wisconsin [7,22]. 
Table 1 Genome-wide target class label assignment to each protein coding gene as a data point for Dickeya didantii 3937 and Pectobacterium carotovorum WPP14

\begin{tabular}{lllllll}
\hline & Total \# CDS* & IF** $^{* *}$ & CF** $^{*}$ & Training data set & Testing data set & Pseudogene \\
\hline Dd3937 & 4520 & 267 & 1264 & 1531 & 2989 & 28 \\
WPP14 & 4590 & 233 & 1111 & 1344 & 3246 & 174 \\
\hline
\end{tabular}

*we only use protein coding genes and pseudogenes are not included.

**IF stands for host-microbe interaction factor; CF stands for genes involved in core biological processes

Colonization and survival in plants requires numerous factors including proteins involved with iron assimilation, protein secretion, exopolysaccharide synthesis, motility, and stress-resistance [23,24]. Five Gene Ontology terms were identified that partition the majority of the positive class training set data into distinct aspects of host-microbe interactions (Table 2). We included all data points in most of our analyses, but also conducted analyses on the partitions defined by these GO annotations (Additional file 1a and $1 \mathrm{~b}$ ). This allows us to test whether different subsystems contain distinct patterns that can be recognized by our learning schemes, while avoiding subsystems with too few genes to provide sufficient information to train the learning schemes.

\section{Assembling training datasets}

The data set for each target genome is assembled separately. Genome sequences, predicted proteins and annotations for both genomes were obtained from the ASAP database [25,26]. Each protein-coding gene in a target genome is considered a data point. The target class label in this specific learning task indicates whether or not a data point has an association with the biological processes involved in host-microbe interaction. A positive class label means the data point is related to hostmicrobe interaction. A negative class label indicates the data point is not likely to be directly involved in host-

Table 2 Ontology for host-microbe interaction, and category assignment genome-wide for data points in Dickeya dadantii (Dd3937) and Pectobacterium carotovora (WPP14)

\begin{tabular}{lrr}
\hline GO term and name & Dd3937 & WPP14 \\
\hline $\begin{array}{l}\text { GO:0052192 movement in environment of other } \\
\text { organism involved in symbiotic interaction; }\end{array}$ & 41 & 41 \\
$\begin{array}{l}\text { GO:0052048 interaction with host via secreted } \\
\text { substance involved in symbiotic interaction }\end{array}$ & 54 & 53 \\
$\begin{array}{l}\text { GO:0051816 acquisition of nutrients from other } \\
\text { organism during symbiotic interaction }\end{array}$ & 103 & 81 \\
GO:0044413 avoidance of host defenses & & \\
GO:0043903 regulation of symbiosis, encompassing \\
mutualism through parasitism \\
* GO:0044403 symbiosis, encompassing mutualism \\
through parasitism
\end{tabular}

*this term is a parent term for all others listed in this table and is used as a generic catch all for host-microbe interaction factors lacking more specific $\mathrm{GO}$ term annotations. microbe interaction, rather it is associated with core biological processes such as transcription and translation or central pathways of metabolism. Positive and negative class labels were assigned by human experts.

For each data point, we assemble a vector of features (or attributes), to characterize it. In our preliminary analyses, we sought to be inclusive in construction of the data matrix. We included 606 attributes for Dd3937 and 598 attributes for WPP14, and these attributes fall roughly into four different categories listed in Table 3. (1) Sequence homology data was obtained from BLASTP searches of the proteins from the target genomes against 239 gamma-proteobacteria from 14 bacterial orders and 58 genomes from other bacterial families outside of gamma-proteobacteria (details in Additional file 2a and 2b). 2) We further summarized sequence homology information by classifying organisms based on phenotypes (e.g.., strict anaerobe), taxonomy (e.g.., the order of Enterobacteriales), habitat (e.g., aquatic), and host type (e.g. plant-associated). Based on this information, we calculated a series of attributes summarizing the homology data. For instance, for each gene, we calculate the number of genomes with a homolog, the fraction of genomes with homologs that are plant-associated, the average similarity scores between homologs, the ratio of the similarity score of plant-associated versus animal-associated homologs, the percentage of hits in the order of Enterobacteriales, and the percentage in facultative anaerobic organisms, etc. Additional file 2c shows the number of genomes in each category used to generate summary attributes. 3) Information related to function and regulation including transcriptome and proteome profiles was incorporated into the attribute vectors (details in Additional file 3a), including microarray experiments with a pecS mutant strain [27], exposure to phenolic acids [28], and growth on potato tuber and stem [29]. For Dd3937, we also integrated the presence of predicted binding sites for 32 transcriptional regulators, including ones related to gene regulation during infection such as PecS [17,27], KdgR [30], H-NS [31,32], and CRP $[33,34]$. We did not include binding site data for WPP14 because the large number of contigs complicates prediction. 4) Finally, we incorporated over 20 basic gene or protein features (Table 3), such as GC content, amino acid composition and computed structural and physiochemical features of proteins and peptides [35], operon prediction 
Table 3 List of all attributes categories used in data set formation in this study, and number of attributes in each categories for all data points in training data set for Dickeya dadantii (Dd3937) and Pectobacterium carotovorum (WPP14)

\begin{tabular}{|c|c|c|c|c|}
\hline Category & Subcategory & Dd3937 & WPP14 & Reference \\
\hline \multirow[t]{3}{*}{ Sequence homology } & Subtotal & 297 & 297 & \\
\hline & Gamma strains & 239 & 239 & Additional file $2 a$ \\
\hline & Non-gamma strains & 58 & 58 & Additional file $2 \mathrm{~b}$ \\
\hline \multirow[t]{3}{*}{ Phenotypes of interest } & Subtotal & 194 & 194 & \\
\hline & Taxonomy Statistics & 76 & 76 & Additional file $2 c, \mathrm{c}$ \\
\hline & Lifestyle Statistics & 118 & 118 & Additional file $2 c$, $\mathrm{c}$ \\
\hline \multirow[t]{12}{*}{ Gene characteristics } & Subtotal & 23 & 21 & \\
\hline & GC content & 1 & 1 & This study \\
\hline & subcellular localization & 1 & 1 & {$[42,46]$} \\
\hline & phylogenetic profile & 6 & 6 & {$[40,41]$} \\
\hline & fingerprints scanning & 3 & 3 & {$[43,44]$} \\
\hline & codon adaptation index (CAI) & 3 & 3 & {$[47,48]$} \\
\hline & physical adjacency (gene neighbor) & 2 & 2 & {$[49,50]$} \\
\hline & Operon prediction & 1 & 1 & {$[36,51]$} \\
\hline & phylogenetic conservation & 1 & 1 & This study \\
\hline & COG functional category & 1 & 1 & [52] \\
\hline & Genomic island & 4 & 1 & {$[53,54]$} \\
\hline & $\begin{array}{l}\text { computed structural and physicochemical } \\
\text { features of proteins and peptides }\end{array}$ & 40 & 66 & {$[35,55]$} \\
\hline \multirow[t]{5}{*}{ Functional genomics } & Subtotal & 52 & 3 & \\
\hline & binding site prediction & 32 & 0 & Additional file $3 b$ \\
\hline & Gene expression & 14 & 3 & Additional file $3 a$ \\
\hline & proteomics & 6 & 0 & Additional file $3 a$ \\
\hline & Total & 606 & 581 & \\
\hline
\end{tabular}

[36], COG functional category [37], and codon adaptation index $[38,39]$. Other gene features are derived from more complex analyses, including: (a) the phylogenetic profile method [40], which is based on the theoretical framework that co-occurrence of functionally linked proteins will be preserved by natural selection [41]; (b) Phylogenetic conservation which classifies genes according to distribution at different branching depths based on our phylogenetic framework for enterobacteria [11]; (c) PSORTb v3.0 [42] which predicts localization as cytoplasmic, cytoplasmic membrane, periplasmic, extracellular, or unknown; (d) Protein fingerprint scanning (a similarity search technique able to identify distantly related proteins) against identified fingerprints associated with virulence factors in PRINTS database [43,44]; and (e) the gene neighbor method which identifies gene physical adjacency on a chromosome [45], based on the theory that neutral evolution tends to shuffle gene orders while functionally associated genes have conserved gene order. We employ both $150 \mathrm{bp}$ and $300 \mathrm{bp}$ as a threshold distance to define gene neighbors using ad hoc code.
Overview of supervised machine learning procedures

The learning procedure is illustrated in Figure 1. (1) First training and testing data sets are assembled by assigning target class labels and forming attribute vectors. (2) Data preprocessing is performed to improve representation and quality, including attribute selection and data transformation, as well as data partitioning according to GO annotations. (3) Both data preprocessing and pattern learning schemes were implemented in Weka package version 3.5.6. [56,57]. Both base and ensemble classifiers were trained to recognize classification patterns. Seven base classifiers were employed in this study including decision tree [58], support vector machine (SVM) using sequential minimal optimization [59-61], Bayesian probabilistic approaches including Bayesian network $[62,63]$ and naive bayes [64], instance based learner $k$ nearest neighbor [65], and propositional rule learner using repeated incremental pruning to produce error reduction (RIPPER) [66]. On top of base classifiers, ensemble classifiers, such as bagging and boosting classifiers, combine multiple models by either sub-sampling a given dataset 


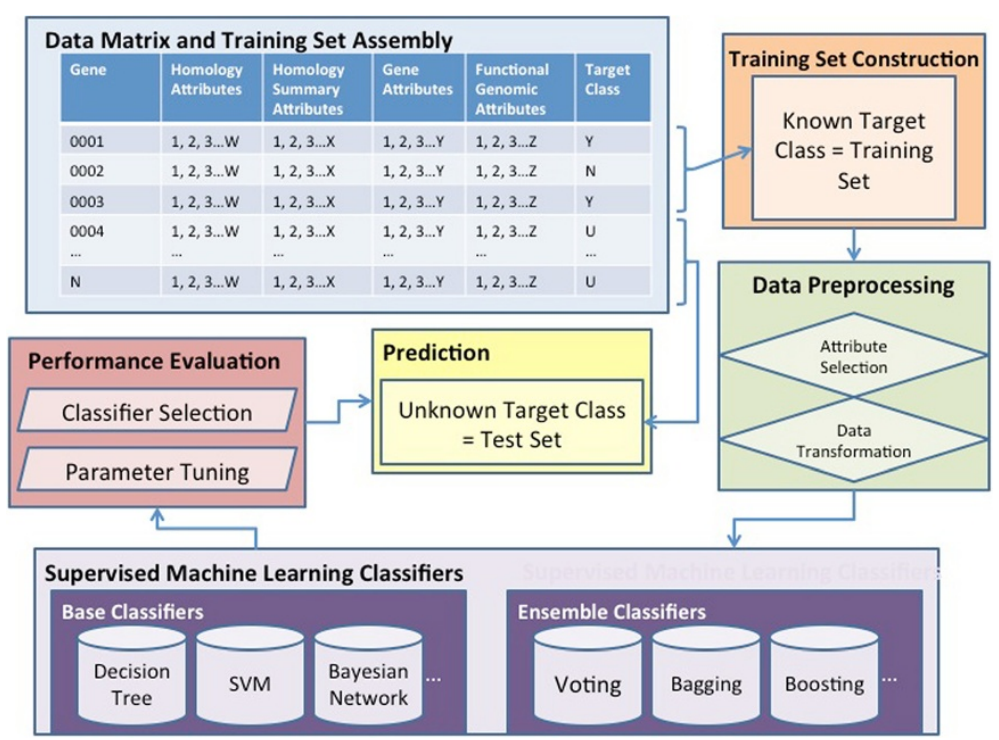

Figure 1 Flow chart of the procedures in performing supervised machine learning tasks of host-microbe interaction factor prediction.

to achieve greater predictive accuracy and reduce overfitting bias [67-70] or combining of probability estimates from different methods [71-73]. Detailed algorithm descriptions and specific settings are described in Additional file 4. (4) Classifier training is followed by classifier performance evaluation, comparison, and selection. Crossvalidation is a technique to assess how accurately a predictive model will perform on an independent data set and whether the model recognizes a pattern that is generalized enough to apply to unseen data $[74,75]$. (5) Based on performance on the training set, we selected the best classifiers to build models and make predictions for the genes that were not part of the training sets.

\section{Data preprocessing}

Attribute extraction, or data transformation, was used to improve the representation of the data sets. Data transformation techniques create extracted attributes from the original attributes, in order to normalize so different attributes are on the same approximate scale, transform all numeric attributes in the dataset to have zero mean and unit variance [76], perform linear mapping of the data to a lower dimensional space in such a way that the variance of the data is maximized using principal components analysis (PCA) [77], or combine attributes where the aggregate feature is more useful than keeping them separate. Since many attributes used in our analysis are continuous data, we also employed data discretization techniques that convert continuous features to discretized or nominal ones to accommodate both data types in the same analysis [78,79]. Another important component in data preprocessing is attribution selection, which is removal of uninformative data since excessive dimensionality can reduce the effectiveness of learning tasks. It includes two steps: an initial clean-up step where the attributes of each type (as listed in Table 3) are tested individually in order to remove the ones with insignificant contribution to classification, which is especially useful for the data types with highest dimensionality. The second step is to evaluate the importance of an attribute passed on from the initial step, and to remove the ones with low importance measurement scores. We used random forest attribute importance measures in this step, which are based on the decrease of classifier performance when values of a variable in a bifurcating tree node are permuted randomly [80], implemented in the extended version of weka 3.5.1 [81,82] (More details in Additional file 4). Furthermore, we performed data decay analysis to define compact attribute sets that maintain informativeness. This involved ranking all attributes based on importance measures from 100 runs using random forest classifiers, gradually decreasing the number of attributes by window size 10 based on their rank, recording the performance of all decayed data sets, and defining the essential set as the point where the overall performance score began to drop.

Evaluating the performance of different learning schemes We used 10-fold cross-validation analyses to evaluate the learned classifiers on random subsets of data withheld from the training sets and averaged across multiple replicates. We recorded a variety of performance statistics for each run including accuracy, true positive rate (TPR or recall), and precision for the positive target class. We also used ROC (Receiver Operating Characteristic) 
curves, PR (Precision-Recall) curves, and the AUC (area under the curve) to evaluate the performance of each constructed classifier. In this particular learning task, we value precision rate as the most important statistic. Precision specifies the proportion of relevant objects being retrieved among all retrieved ones, a factor that is particularly important to define a candidate list with high confidence for downstream experimental validation. On the other hand, recall is the proportion of relevant objects that are retrieved. When a situation does not allow both precision and recall rates to be high at the same time, we give the precision rate precedence over the recall rate. ROC and PR curves are widely regarded as more appropriate than any individual statistic in evaluating classification algorithms [83]. A ROC curve is a graphical technique that plots the correlation of correctly classified data points with falsely classified ones, in order to characterize the tradeoff between true positive and false positive rates. PR curves depict the correlation of how precisely the algorithm identifies the data points in their class with how many "true" data points are retrieved and provide a good complement to ROC curves which can be overly optimistic [84].

\section{Results and discussion}

Many computational methods have been used to identify gene functions involved in host-microbe interaction, and most of them rely primarily on homology-based searches using known interaction determinants as bait to identify new candidate genes. These methods are often successful, but neglect many genes of unknown function and strain/clade-specific genes, which could play an important role in host-microbe interactions and bacterial niche adaptations $[85,86]$. Overcoming these limitations with the current methodologies is critical to expanding our understanding of the complex molecular mechanisms underlying host-microbe interactions. The value of machine learning not only lies in deriving knowledge based on pattern recognition, but also providing an automated alternative to having a human expert repeatedly sift through large and complex datasets.

\section{Some attributes are more useful than others to predict host-microbe interaction}

Our results indicate that although all categories outperform randomized data, different major categories of attributes contribute differently to learning scheme performance as shown in the ROC curve for Dd3937 in Figure 2 and Additional file 4. Gene features and summarized homology information were most useful in classifying hostmicrobe interaction factors, while data related to computed structural or physiochemical characteristics, and gene functionality data, including gene expression, binding site predictions, and proteomics profiles, performed less well. Further analysis of the gene functionality attributes using random forest importance measurement scores indicates that the data corresponding to many of these attributes are relatively noisy and do not correlate well with the target class, though a subset, such as KdgR binding site predictions, do correlate well. Some of our attributes are themselves the results of other pattern recognition methods. For example, phylogenetic profiles, one of the most useful attributes, are based on an unsupervised learning approach, where no prior information is given to the learner regarding the output or class label. Our

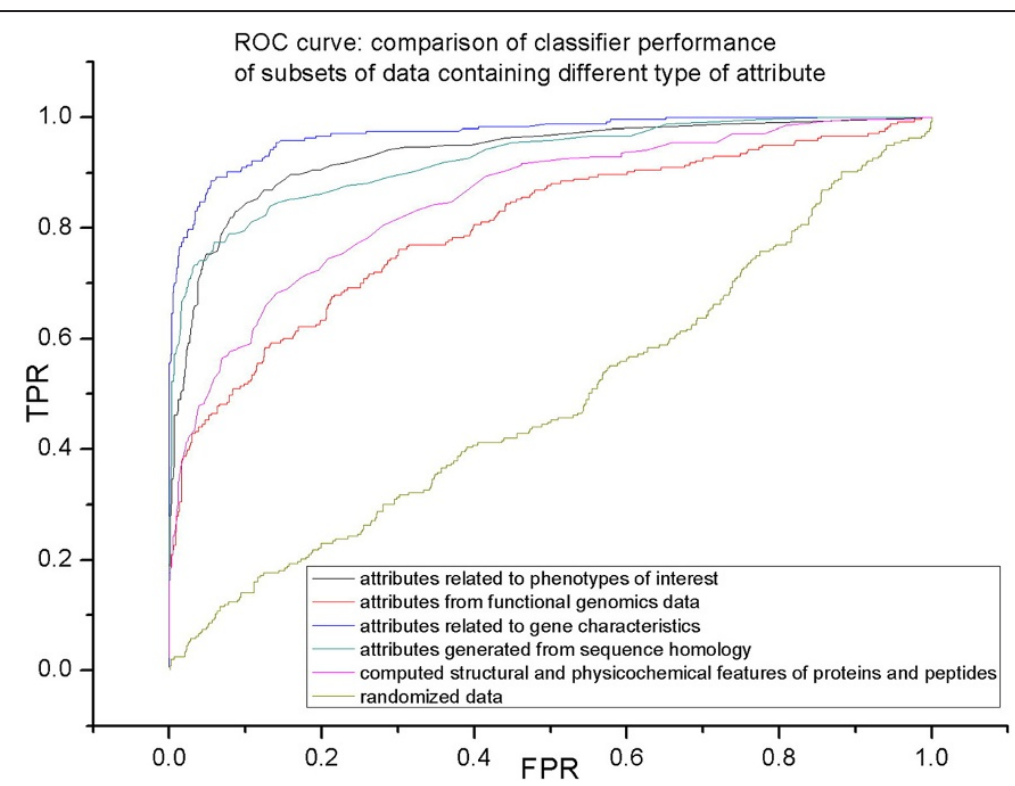

Figure 2 ROC curve to compare classifier performance of different data sets containing various types of attributes as listed in Table 3. (TPR: True Positive Rate; FPR: False Positive Rate). 
analysis is a good example of how supervised and unsupervised learning algorithms can be combined to make better inference.

We conducted data decay analysis to obtain additional insight into the most informative attributes. The size of the final compact attribute sets is 45 and 31 for Dd3937 and WPP14, respectively, as shown in Additional file 5b. The majorities of attributes in the compact sets are summaries of homology data according to phenotypes or computed gene features, and many of the retained attributes are shared between both strains despite the independent machine learning analyses. The common list includes five gene feature attributes including phylogenetic profile, gene cluster from operon prediction, gene neighbor, cellular localization, and amino acid composition. The most informative homology attributes include percentage, average value, or sum value of a given gene having homologous hits with organisms having different pathogenicity and habitat phenotypes. In addition, the homology data summarized by phenotypes related to growth condition and taxonomic groups is also informative including having homologs in anaerobic organisms, facultative anaerobes and their ratio, and having homologs in other gamma-proteobacteria, and enterobacteria, all of which appear in the selected attribute list for both strains.

Overall, these results suggest that attributes which are relatively simple to assemble from standard BLASTP searches, coupled with a handful of additional easily computed features are sufficient to achieve good performance in this machine learning task. This is particularly encouraging for development of a generalized approach for future applications to predict host-interaction factors across a broad range of bacterial phytopathogens.

\section{Preprocessing and partitioning can improve performance}

The PR curve shown in Figure 3 illustrates the improvement in performance that we achieved through attribute selection, data discretization, and data partitioning according to GO terms. 1) Attribute selection generates more cost-effective learning schemes by reducing data set dimensionality by removing uninformative attributes, in order to improve the overall performance of the learning schemes $[87,88]$. After benchmarking different attribute selection techniques such as filter (e.g., subset attribute selection [89]) and wrapper methods (e.g., Naive Bayes with forward selection algorithm) as well as attribute ranking (e.g., SVM Attribute evaluator [90] and information gain), we chose random forest importance measures in this study because it is robust to noise, relatively computationally efficient, and is suitable for data sets with high dimensionality hence reducing the risk of overfitting [81]. After feature selection, our data sets contain 105 and 122 attributes, which are $17.3 \%$ and $21 \%$ of the original data size of Dd3937 and WPP14, respectively. 2) By comparing different data transformation techniques (Additional file 6a), supervised data discretization was shown to be substantially better for improving classifier performance than other methods. Supervised discretization techniques are

RP curve: evaluation of different strategies to boost classifier performance

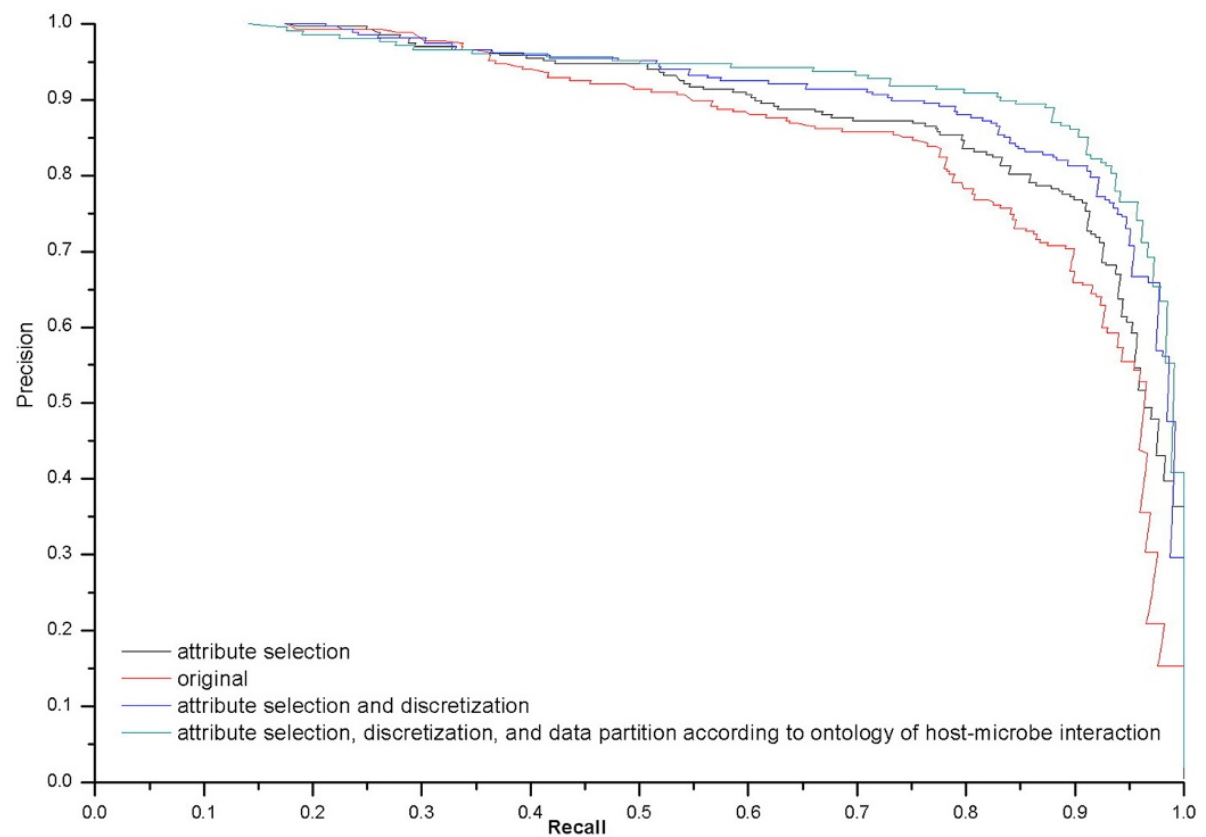

Figure 3 PR (Precision-Recall) curve to evaluate strategies for boosting classifier performance. 
suitable for high dimensional data as they significantly reduce the number of possible values of continuous features, and also discretize an attribute according to its class label $[91,92] .3)$ We also saw an improvement when we coupled the preprocessing with partitioning the learning task into several separate tasks based on assigning genes in the training set according to GO terms. This result suggests that some subsystems, such as localization in host and secretion of host interaction proteins, are substantially more informative and suitable for our learning task (Additional file 6b). Other subsystems, such interaction with host defense systems and transcriptional regulation of host interaction genes, performed less convincingly, possibly because these subsystems are involved in host-microbe interaction but also include other genes not implicated in this biological process. For example, the global DNAbinding regulator hns gene also modulates flagella genes and lipopolysaccharide production that are important for initial bacterial attachment to host cell surfaces $[93,94]$. These data points were removed from subsequent analysis. Our result suggests that our learning schemes hold predictive power for the subsystems involved with complex biological processes during host-microbe interaction, but do not accurately distinguish the patterns for some subsystems that are closely intertwined with other cellular processes.

\section{The performance of machine learning schemes is statistically encouraging}

In this study, we employed several strategies to mitigate the potential overfitting issues that are important for effective supervised machine learning tasks. Simply put, overfitting occurs when the predictive model learns a pattern that is overly specific to the training data but not

Table 4 Statistics for positive class object prediction and parameters used in selected learning schemes for both Dickeya dadantii 3937 and Pectobacterium carotovorum WPP14

\begin{tabular}{|c|c|c|c|c|c|c|}
\hline Classifiers & Precision & TPR/recall/sensitivity & specificity/TNR & accuracy & F-measure & AUC \\
\hline \multicolumn{7}{|l|}{ Dd3937 } \\
\hline Random Forest & 0.93 & 0.81 & 0.98 & 0.94 & 0.87 & 0.97 \\
\hline Bayesian Network & 0.91 & 0.85 & 0.97 & 0.94 & 0.88 & 0.97 \\
\hline SMO using RBF kernels & 0.93 & 0.85 & 0.98 & 0.95 & 0.89 & 0.92 \\
\hline SMO using polynormial kernels & 0.91 & 0.87 & 0.97 & 0.95 & 0.95 & 0.89 \\
\hline Adaptive Boosting (Naïve Bayes)* & 0.84 & 0.89 & 0.95 & 0.93 & 0.87 & 0.96 \\
\hline Adaptive Boosting (Decision Tree)* & 0.96 & 0.91 & 0.99 & 0.97 & 0.93 & 0.98 \\
\hline Adaptive Boosting (IBK)* & 0.96 & 0.84 & 0.99 & 0.95 & 0.90 & 0.99 \\
\hline Adaptive Boosting (Decision Stump)* & 0.92 & 0.87 & 0.98 & 0.95 & 0.89 & 0.97 \\
\hline Multi-Boosting (Decision Tree)* & 0.97 & 0.91 & 0.99 & 0.97 & 0.94 & 0.98 \\
\hline Multi-Boosting (IBK)* & 0.91 & 0.77 & 0.98 & 0.93 & 0.84 & 0.93 \\
\hline Multi-Boosting (Naïve Bayes)* & 0.90 & 0.91 & 0.97 & 0.95 & 0.91 & 0.96 \\
\hline Logit-Boosting (Decision Stump)* & 0.91 & 0.90 & 0.97 & 0.96 & 0.91 & 0.98 \\
\hline \multicolumn{7}{|l|}{ WPP14 } \\
\hline Random Forest & 0.89 & 0.81 & 0.97 & 0.93 & 0.85 & 0.97 \\
\hline Bayesian Network & 0.90 & 0.83 & 0.97 & 0.94 & 0.87 & 0.97 \\
\hline SMO using RBF kernels & 0.94 & 0.84 & 0.98 & 0.95 & 0.89 & 0.91 \\
\hline SMO using polynormial kernels & 0.93 & 0.86 & 0.98 & 0.95 & 0.95 & 0.89 \\
\hline Adaptive Boosting (Naïve Bayes)* & 0.89 & 0.89 & 0.97 & 0.95 & 0.89 & 0.96 \\
\hline Adaptive Boosting (Decision Tree)* & 0.95 & 0.86 & 0.99 & 0.96 & 0.90 & 0.98 \\
\hline Adaptive Boosting (IBK)* & 0.87 & 0.83 & 0.96 & 0.93 & 0.85 & 0.92 \\
\hline Logit-Boosting (Decision Stump)* & 0.90 & 0.85 & 0.97 & 0.94 & 0.88 & 0.97 \\
\hline Multi-Boosting (Decision Tree)* & 0.94 & 0.86 & 0.98 & 0.96 & 0.90 & 0.98 \\
\hline Multi-Boosting (Decision Stump)* & 0.91 & 0.75 & 0.98 & 0.93 & 0.82 & 0.97 \\
\hline Multi-Boosting (Naïve Bayes)* & 0.90 & 0.89 & 0.97 & 0.95 & 0.89 & 0.96 \\
\hline Logit-Boosting (Decision Stump)* & 0.90 & 0.87 & 0.97 & 0.95 & 0.89 & 0.97 \\
\hline
\end{tabular}

*: denote ensemble classifiers, with base learner being shown within parenthesis. 
generalized enough to perform equally well on unseen data [95]. We strived to maximize inclusion of relevant attributes to mitigate the problem of overfitting to increase model replicability [96], while excluding unimportant attributes that may be detrimental to pattern recognition schemes performance. Additionally, we hold out pristine examples for testing, integrated result over multiple classifiers retaining only predictions that show a high degree of consensus, chose classifier parameters based on the cross-validation tests, and used a simpler predictor where possible, to address the overfitting issue.

Overall the results of using supervised machine learning schemes on host-microbe interaction factor prediction are statistically encouraging, achieving over $84 \%$ precision rate and $75 \%$ recall rate from 10 -fold cross validation evaluation. We used a nested 10-fold cross-validation that includes an "outer" 10-round cross-validation, which averages data variability from 10 different data partitions. Each data partition sets aside $10 \%$ of the data set (outer test set) to measure the performance of the predictive model generated from the other $90 \%$ of the data (outer training set). Each outer training set is used to choose the value of tuning parameters for this model in order to achieve optimal performance. The parameter-tuning step is especially important for SVM and K-nearest neighbor learning schemes which are particularly sensitive to parameter settings (Stone 1977). Performance
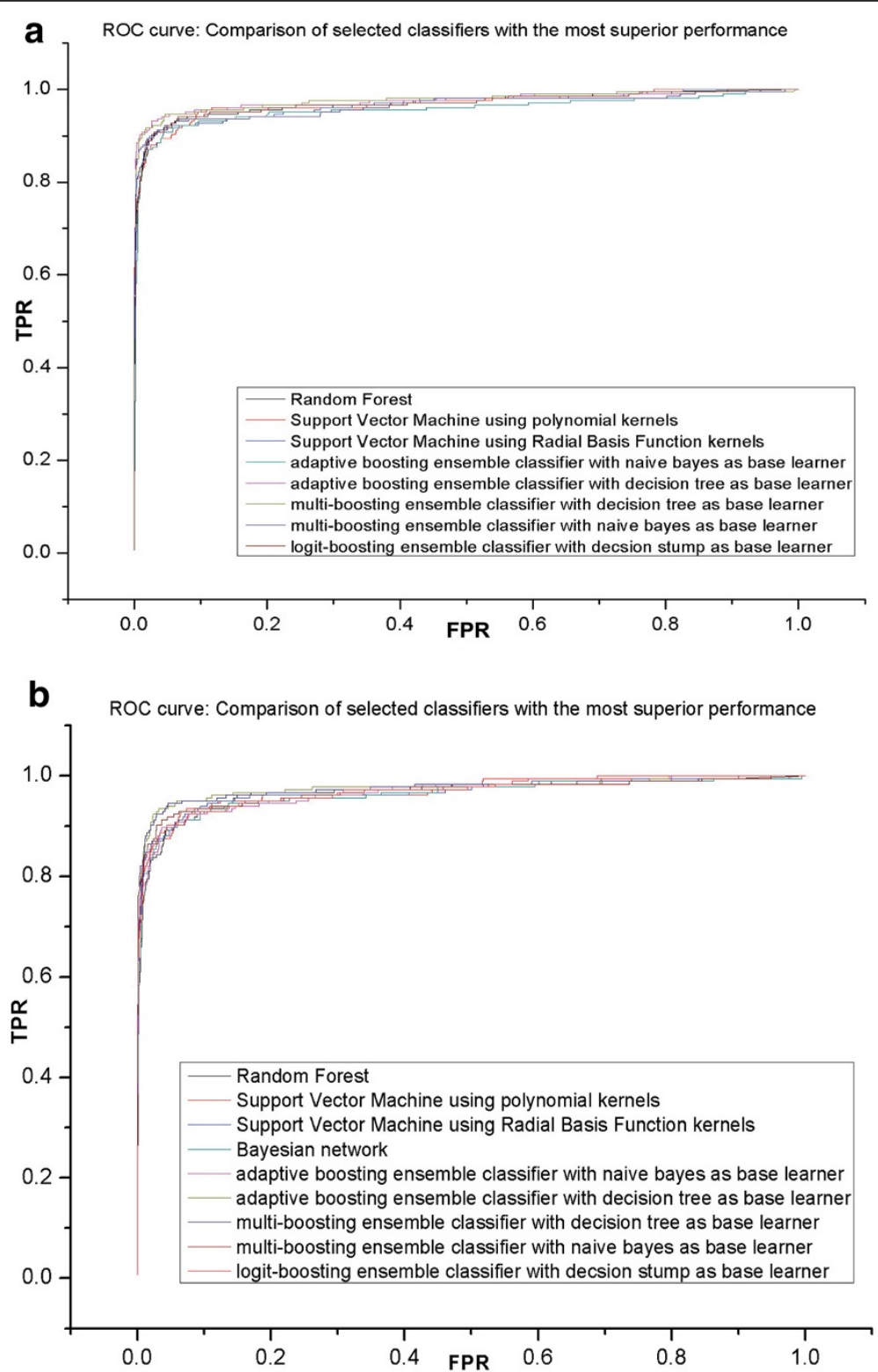

Figure 4 Comparison of the selected learning schemes. (a) ROC curve for Dickeya dadantii 3937, (b) ROC curve for Pectobacterium carotovorum WPP14. (TPR: True Positive Rate; FPR: False Positive Rate). 
Table 5 Top 50 predicted host-microbe interaction factors from Dickeya dadantii 3937

\begin{tabular}{|c|c|c|c|}
\hline FeatureID & Prob & Name & Annotation \\
\hline ABF-0018715 & 0.922 & virB8 & $\begin{array}{l}\text { Inner membrane protein forms channel for type IV secretion } \\
\text { of T-DNA complex (VirB8) }\end{array}$ \\
\hline ABF-0020188 & 0.922 & & $\begin{array}{l}\text { Predicted cell-wall-anchored protein SasA (LPXTG motif) this } \\
\text { is up-regulated by hrpY; we have a mutation in this gene. }\end{array}$ \\
\hline ABF-0019950 & 0.922 & & Putative multicopper oxidase \\
\hline ABF-0019360 & 0.922 & & hypothetical protein \\
\hline ABF-0019151 & 0.922 & & chrysobactin synthetase cbsF \\
\hline ABF-0019124 & 0.922 & & Biopolymer transport protein ExbD/TolR \\
\hline ABF-0019122 & 0.922 & & MotA/TolQ/ExbB proton channel family protein \\
\hline ABF-0019117 & 0.922 & sftP & TonB-dependent receptor \\
\hline ABF-0019116 & 0.922 & & hypothetical protein \\
\hline ABF-0018783 & 0.922 & & putative transmembrane protein \\
\hline ABF-0018775 & 0.922 & & Holin \\
\hline ABF-0018724 & 0.922 & & putative ATP/GTP-binding protein remnant \\
\hline ABF-0018722 & 0.922 & virB2 & Major pilus subunit of type IV secretion complex (VirB2) \\
\hline ABF-0047137 & 0.922 & & hypothetical protein \\
\hline ABF-0018717 & 0.922 & virB6 & $\begin{array}{l}\text { Integral inner membrane protein of type IV secretion } \\
\text { complex (VirB6) }\end{array}$ \\
\hline ABF-0018716 & 0.922 & virB7 & TriF protein \\
\hline ABF-0018713 & 0.922 & virB10 & $\begin{array}{l}\text { Inner membrane protein forms channel for type IV secretion } \\
\text { of T-DNA complex (VirB10) }\end{array}$ \\
\hline ABF-0018712 & 0.922 & virB11 & $\begin{array}{l}\text { ATPase provides energy for both assembly of type IV secretion } \\
\text { complex and secretion } \\
\text { of T-DNA complex (VirB11) }\end{array}$ \\
\hline ABF-0018601 & 0.922 & & hypothetical protein \\
\hline ABF-0018207 & 0.922 & & hypothetical protein \\
\hline ABF-0018199 & 0.922 & ganC & putative truncated PTS system EIIBC component \\
\hline ABF-0017777 & 0.922 & hecA2 & Putative member of ShIA/HecA/FhaA exoprotein family \\
\hline ABF-0015606 & 0.922 & & $A B C$ transporter permease protein \\
\hline ABF-0015604 & 0.922 & & $\begin{array}{l}\text { Amino acid } A B C \text { transporter, periplasmic amino acid-binding } \\
\text { protein }\end{array}$ \\
\hline ABF-0015543 & 0.922 & & $\begin{array}{l}\text { hypothetical protein } 15544 \text { is up-regulated by hrpY. Is } 15543 \\
\text { in the same operon? } \\
\text { We have a mutation in } 15544\end{array}$ \\
\hline ABF-0015387 & 0.922 & nipE & necrosis-inducing protein \\
\hline ABF-0014838 & 0.922 & & putative exported protein \\
\hline ABF-0014623 & 0.922 & & Type IV pilus biogenesis protein PilN \\
\hline ABF-0018720 & 0.922 & virB4 & $\begin{array}{l}\text { ATPase provides energy for both assembly of type IV secretion } \\
\text { complex and secretion } \\
\text { of T-DNA complex (VirB4) }\end{array}$ \\
\hline ABF-0018714 & 0.922 & virB9 & VirB9 \\
\hline ABF-0047204 & 0.922 & & hypothetical protein \\
\hline ABF-0015913 & 0.921 & ppdA & Prepilin peptidase dependent protein A \\
\hline ABF-0017252 & 0.921 & & Conjugative transfer protein TrbG \\
\hline ABF-0018195 & 0.921 & ganG & galactan $A B C$ transport system, permease component \\
\hline ABF-0016407 & 0.921 & & hypothetical protein \\
\hline ABF-0018205 & 0.921 & & Pirin \\
\hline ABF-0019418 & 0.921 & & Cellulose 1, 4-beta-cellobiosidase precursor \\
\hline
\end{tabular}


Table 5 Top 50 predicted host-microbe interaction factors from Dickeya dadantii 3937 (Continued)

\begin{tabular}{|c|c|c|c|}
\hline ABF-0019468 & 0.921 & & hypothetical protein \\
\hline ABF-0019566 & 0.921 & & hypothetical protein \\
\hline ABF-0016680 & 0.921 & & Iron utilization protein \\
\hline ABF-0020727 & 0.921 & sttG & General secretion pathway protein $\mathrm{G}$ \\
\hline ABF-0019115 & 0.921 & & hypothetical protein \\
\hline ABF-0015381 & 0.921 & avrM & Avirulence protein \\
\hline ABF-0018723 & 0.921 & virB1 & VirB1 \\
\hline ABF-0015598 & 0.921 & & hypothetical protein \\
\hline ABF-0015609 & 0.921 & & Branched-chain amino acid aminotransferase \\
\hline ABF-0018193 & 0.921 & ganF & galactan ABC transport system, permease component \\
\hline ABF-0017097 & 0.921 & & Methyl-accepting chemotaxis protein \\
\hline ABF-0020433 & 0.921 & & hypothetical protein \\
\hline ABF-0019153 & 0.921 & $\mathrm{cbsH}$ & chrysobactin oligopeptidase $\mathrm{CbsH}$ \\
\hline
\end{tabular}

statistics for different classifiers are listed in Table 4, excluding classifiers with precision rates $<80 \%$. ROC curves of selected classifiers for WPP14 are shown in Figure 4.

The comparison of base classifier performances indicates SVM and random forest outperforms other base classifiers (data not shown), and ensemble classifiers generally perform better than base classifiers, especially the boosting algorithms using decision trees as the base learner. The ensemble classifiers integrate results over multiple classifiers in order to average out the "classifier effect”. For example, some classifiers such as Naïve Bayes can be overly optimistic with a lower precision rate [97], and adaptive boosting ensemble classifiers with Naïve Bayes as the base learner can optimize precision and total accuracy rate through incrementally iterative learning processes [98]. The performance curves of selected classifiers are shown in Figure 4a and 4b for Dd3937 and WPP14 respectively. The best performing classifier for $\operatorname{Dd} 3937$ is the adaptive boosting ensemble classifier [70] with decision trees as the base learner, which achieved a precision rate above $97 \%$ with over $87 \%$ recall rate. The best performing classifier for WPP14 is the multi-boosting ensemble classifier [69] with decision trees as the base learner, which reached a precision above $94 \%$ with over $82 \%$ recall rate. Using the constructed predictive models from selected classifiers, we are able to make predictions for data points with previously unknown relation to host-microbe interactions.

\section{A significantly extended list of host-microbe interaction factors is revealed}

Application of different learned classifiers to the target genomes as a whole allows us to generate a conservative set of predictions for downstream experimentation. We pay the most attention to precision to ensure the retrieved data points are most relevant to host-microbe interaction to facilitate subsequent experimental validation. In order to call a gene a "predicted host-interaction factor", we required strict consensus across the different classifiers with an average precision score in excess of thresholds defined by the ROC curves (92\% and 89\% for Dd3937 and WPP14, respectively). The selected classifiers generally agree with each other, and about two thirds of all unknown genes are unanimously predicted by all classifiers to be either host-microbe interaction factors or genes involved in core biological processes. Using these criteria, a total of 1726 genes (57.7\% of Dd3937 genes) in Dd3937 and 2180 genes (67.2\% of WPP14 genes) in WPP14 are predicted not to involved in host-microbe interactions. There are 211 genes (7.1\% of Dd3937 genes) in Dd3937 and 216 genes (6.7\% of WPP14 genes) in WPP14 classified as putative interaction factors. The remaining 1052 genes (35.1\% of Dd3937 genes) and 850 genes (26.2\% of WPP14 genes) are left as unclassified. The top 50 predicted host-microbe interaction factors for Dickeya dadantii 3937 and Pectobacterium carotovorum WPP14 are listed in Tables 5, 6 and 7, and the entire list of predicted host-microbe interaction factors for both strains are in Additional file $7 \mathrm{a}$ and $7 \mathrm{~b}$. These lists partially overlap, with 56 orthologs identified as interaction factors in both organisms. Given the phylogenetic relationship between these two phytopathogens and the similarity of their pathogenic phenotypes, we did expect this result; however, the learning tasks were executed independently and agreement across organisms was not a given.

One striking observation is the large number of genes of unknown function from the predicted list of hostmicrobe interaction factors. Among all predicted interaction factors, over $30 \%$ of them currently have no or very little annotated information, and many of them are ORFans [99-102] without any homolog to 297 bacterial genomes inspected. Among the 56 genes found in 
Table 6 Top 50 predicted host-microbe interaction factors from Pectobacterium carotovorum WPP14

\begin{tabular}{|c|c|c|c|}
\hline ID & Prob & Name & Product \\
\hline ADT-0001591 & 0.912 & & hypothetical protein \\
\hline ADT-0003750 & 0.912 & & putative exported protein \\
\hline ADT-0000805 & 0.912 & dltB & peptidoglycan biosynthesis protein \\
\hline ADT-0003928 & 0.911 & & pectate lyase \\
\hline ADT-0003247 & 0.911 & & methyl-accepting chemotaxis protein \\
\hline ADT-0000806 & 0.911 & $\mathrm{dltD}$ & $\begin{array}{l}\text { poly(glycerophosphate chain) D-alanine } \\
\text { transfer protein }\end{array}$ \\
\hline
\end{tabular}

ADT-0003745 0.911

ABC transporter ATP binding protein

ADT-0002063 0.911

ADT-0000400 0.911 hasE

ADT-0003089 0.911

ADT-0003418 0.910

ADT-0000941 0.910

ADT-0006368 0.910

ADT-0005582 0.910

ADT-0000983 0.910

ADT-0001252 0.910

ADT-0003245 0.910

ADT-0000027 0.910

ADT-0003542 0.909

ADT-0001195 0.909

ADT-0004315 0.909 astB

ADT-0003152 0.909

ADT-0002357 0.908

ADT-0000543 0.908

ADT-0001392 0.908

ADT-0002087 0.908

ADT-0001868 0.908

ADT-0000803 0.908

ADT-0000571 0.908

ADT-0000535 0.908

ADT-0001404 0.907

ADT-0004320 $0.907 \quad \mathrm{sftP}$

ADT-0001744 0.907

ADT-0003391 0.907

ADT-0003535 0.907

ADT-0003563 0.907

ADT-0001980 0.907

ADT-0000804 0.907 dltA

ADT-0001616 0.906

ADT-0001394 0.906 hypothetical protein

HlyD family secretion protein

$\mathrm{N}$-terminal fragment of a diguanylate cyclase (pseudogene)

methyl-accepting chemotaxis protein

methyl-accepting chemotaxis protein

hypothetical protein

hypothetical protein

methyl-accepting chemotaxis protein

$A B C$ transporter permease protein

methyl-accepting chemotaxis protein

methyl-accepting chemotaxis protein

putative type IV pilus protein

LysR-family transcriptional regulator

sulfate ester $A B C$ transporter permease protein

methyl-accepting chemotaxis protein methyl-accepting chemotaxis protein

$A B C$ transporter, substrate binding protein putative exported protein

putative signaling protein

LysR-family transcriptional regulator

acyl carrier protein

putative cellulase

putative lipoprotein

hypothetical protein

TonB-dependent receptor

putative exported protein

putative membrane protein

hypothetical protein

LysR-family transcriptional regulator

hypothetical protein

putative D-alanine--poly(phosphoribitol) ligase subunit 1

putative transport system membrane protein

hypothetical protein
Table 6 Top 50 predicted host-microbe interaction factors from Pectobacterium carotovorum WPP14 (Continued)

\begin{tabular}{|c|c|c|}
\hline ADT-0001320 & 0.906 & methyl-accepting chemotaxis protein \\
\hline ADT-0001567 & 0.906 & putative exported protein \\
\hline ADT-0005614 & 0.906 & hypothetical protein \\
\hline ADT-0001436 & 0.906 & $\begin{array}{l}\text { putative component of polysulfide } \\
\text { reductase }\end{array}$ \\
\hline ADT-0004253 & 0.906 occQ & $\begin{array}{l}\text { octopine transport system permease } \\
\text { protein }\end{array}$ \\
\hline ADT-0001493 & 0.905 & hypothetical protein \\
\hline ADT-0001492 & 0.905 & putative lipoprotein \\
\hline ADT-0002704 & 0.905 & putative lipoprotein \\
\hline ADT-0002584 & 0.905 & $\begin{array}{l}\text { ABC transporter, membrane spanning } \\
\text { protein }\end{array}$ \\
\hline
\end{tabular}

interaction factor lists for both strains, roughly one third have no clear functional assignment. 13 hypothetical proteins in both strain lists are "unknown unknowns", a term used to indicate there is no information at all available for that gene [103]. The other 9 of them are so-called "known unknown" proteins, meaning they only have information in general biological terms, such as putative exported protein, putative transmembrane protein, and probable lipoprotein. This result suggests a substantial portion of the genome cannot be screened using conventional similarity-based searches, and our more sophisticated pattern recognition approach was able to identify candidate interaction factors that would be missed using homology-based methods.

The remaining two-thirds of predicted interaction factors are annotated with various (at least partially) informative functions. The lists include genes with previously characterized roles in host-microbe interaction in these or very closely related organisms that were overlooked by the human experts who assembled the training set. For example, Dd3937 secretes plant cell wall degrading enzymes through a type II secretion system for plant host cell wall degradation in turn using the released nutrients as carbon sources for growth [104], and a group of genes related to this process are predicted with high confidence including predicted proteins previously reported to play an accessory role in utilization of galactose, a major component of pectin, in Dd3937 [105]. A knockout mutant of a necrosisinducing protein included in the prediction list has been experimentally shown to have reduced virulence in a Pectobacterium strain [106]. Further, our lists also include genes with homologs implicated in host-microbe interaction in more distantly related organisms. There are 9 genes that were shown with direct or indirect evidence to be involved with metal homeostasis in different bacteria, including $\operatorname{exb} B$, exbD, and ton $B$ genes which are essential for ferric iron uptake in Escherichia coli [107], Xanthomonas campestris [108], Pseudomonas putida [109], and Photorhabdus 
Table 7 List of 56 genes predicted host-microbe interaction factors in both Dickeya dadantii 3937 and Pectobacterium carotovorum WPP14

\begin{tabular}{|c|c|c|c|c|c|}
\hline \multicolumn{3}{|l|}{ Dd3937 } & \multicolumn{3}{|l|}{ WPP14 } \\
\hline FeatureID & Name & Product & FeatureID & Name & Product \\
\hline ABF-0019117 & sftP & TonB-dependent receptor & ADT-0004320 & sftP & TonB-dependent receptor \\
\hline ABF-0019116 & & hypothetical protein & ADT-0004318 & & unknown \\
\hline ABF-0018207 & & hypothetical protein & ADT-0001980 & & hypothetical protein \\
\hline ABF-0015604 & & Amino acid $A B C$ transporter & ADT-0000748 & & putative extracellular solute-binding protein \\
\hline ABF-0015387 & nipE & necrosis-inducing protein & ADT-0000781 & & putative exported protein \\
\hline ABF-0014838 & & putative exported protein & ADT-0002655 & & putative exported protein \\
\hline ABF-0019124 & & Biopolymer transport protein ExbD/TolR & ADT-0002263 & & putative biopolymer transport protein \\
\hline ABF-0019115 & & hypothetical protein & ADT-0002265 & & hypothetical protein \\
\hline ABF-0017097 & & Methyl-accepting chemotaxis protein & ADT-0003418 & & methyl-accepting chemotaxis protein \\
\hline ABF-0019566 & & hypothetical protein & ADT-0001832 & & putative exported protein \\
\hline ABF-0016407 & & hypothetical protein & ADT-0001404 & & hypothetical protein \\
\hline ABF-0015906 & & 6-phosphogluconolactonase & ADT-0003106 & & putative exported protein \\
\hline ABF-0019118 & atsR & Alkanesulfonates-binding protein & ADT-0001174 & atsR & putative sulfate ester binding protein \\
\hline ABF-0019125 & astB & $\begin{array}{l}\text { Alkanesulfonates transport system } \\
\text { permease protein }\end{array}$ & ADT-0004315 & astB & sulfate ester $A B C$ transporter permease protein \\
\hline ABF-0017125 & inh & Alkaline proteinase inhibitor precursor & ADT-0001911 & inh & protease inhibitor \\
\hline ABF-0019002 & & hypothetical protein & ADT-0001744 & & putative exported protein \\
\hline ABF-0019205 & & $A B C$ transporter & ADT-0002584 & & $\mathrm{ABC}$ transporter \\
\hline ABF-0014642 & & hypothetical protein & ADT-0000571 & & putative cellulase \\
\hline ABF-0019092 & & Transcriptional activator protein lysR & ADT-0001195 & & LysR-family transcriptional regulator \\
\hline ABF-0016585 & & Methyl-accepting chemotaxis protein & ADT-0001320 & & methyl-accepting chemotaxis protein \\
\hline ABF-0019383 & & D-alanyl transfer protein DltB & ADT-0000805 & dltB & peptidoglycan biosynthesis protein \\
\hline ABF-0019855 & & $\begin{array}{l}\text { Methyl-accepting chemotaxis protein II } \\
\text { (aspartate chemoreceptor protein) }\end{array}$ & ADT-0001887 & & putative methyl-accepting chemotaxis protein \\
\hline ABF-0015168 & $\operatorname{chm} X$ & $\begin{array}{l}\text { Methyl-accepting chemotaxis protein III } \\
\text { (ribose and galactose chemoreceptor protein) }\end{array}$ & ADT-0003152 & & methyl-accepting chemotaxis protein \\
\hline ABF-0018737 & & DNA-binding protein & ADT-0003335 & & putative regulatory protein \\
\hline ABF-0019933 & & hypothetical protein & ADT-0003354 & & hypothetical protein \\
\hline ABF-0014645 & & Paraquat-inducible protein $\mathrm{A}$ & ADT-0002701 & & putative membrane protein \\
\hline ABF-0017674 & & Methyl-accepting chemotaxis protein & ADT-0003245 & & methyl-accepting chemotaxis protein \\
\hline ABF-0020681 & & hypothetical protein & ADT-0002418 & & RES domain-containing protein \\
\hline ABF-0015907 & & TonB-dependent hemin & ADT-0002398 & & TonB-dependent hemin \\
\hline ABF-0018934 & & 4-aminobutyrate aminotransferase & ADT-0002845 & & putative class-III aminotransferase \\
\hline ABF-0014824 & & $\begin{array}{l}\text { Methyl-accepting chemotaxis protein II } \\
\text { (aspartate chemoreceptor protein) }\end{array}$ & ADT-0002104 & & methyl-accepting chemotaxis protein \\
\hline ABF-0018178 & & Iron(III) dicitrate-binding protein & ADT-0002009 & & $\begin{array}{l}\text { putative periplasmic substrate-binding } \\
\text { transport protein }\end{array}$ \\
\hline ABF-0019391 & & Pectate lyase & ADT-0003928 & & pectate lyase \\
\hline ABF-0015887 & & hypothetical protein & ADT-0002063 & & hypothetical protein \\
\hline ABF-0016115 & & Methyl-accepting chemotaxis protein & ADT-0000027 & & methyl-accepting chemotaxis protein \\
\hline ABF-0019101 & atsB & $\begin{array}{l}\text { Alkanesulfonates transport system permease } \\
\text { protein }\end{array}$ & ADT-0003749 & atsB & putative sulfate ester transporter \\
\hline ABF-0019214 & & Glucosamine kinase GpsK & ADT-0003604 & & hypothetical protein \\
\hline ABF-0016752 & & Ferric siderophore transport system & ADT-0003559 & & TonB-like protein \\
\hline
\end{tabular}




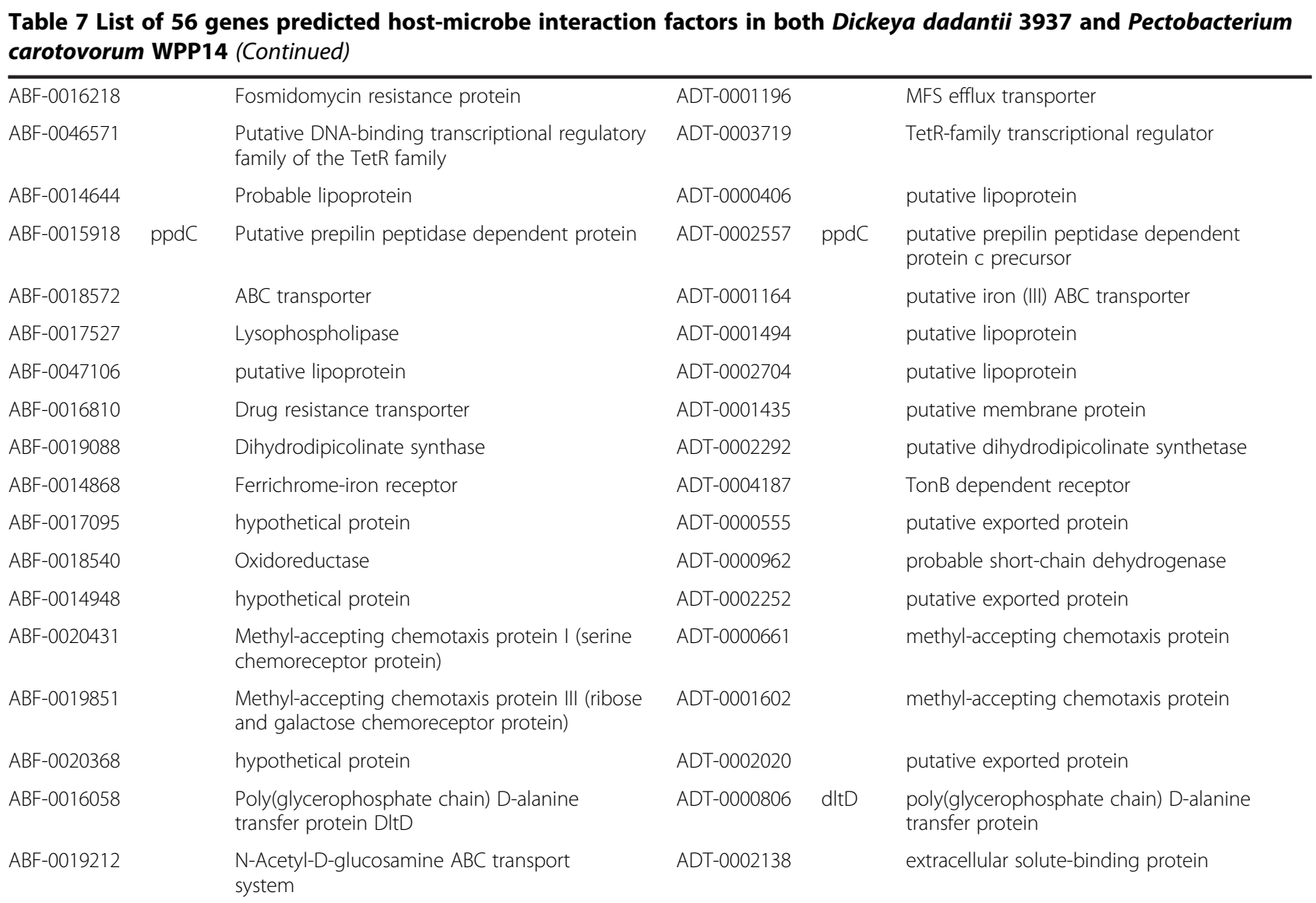

temperate [110], as well as ferric siderophore transporter and ferrichrome-iron receptor genes, and a cytochrome b gene $(c y b C)$ that is positively regulated by Fur and others that encode iron-dependent proteins in Salmonella enterica [111]. The predicted lists also include orthologs of the $d l t B$ gene implicated in cell surface adhesion in Staphylococcus aureus [112], the srfA gene that encodes secreted effect or protein in Pantoea ananatis [113], a LysR-family regulator associated with quorum sensing in Pseudomonas aeruginosa [114], the cell-wall-anchored protein SasA suggested to play a role in adhesion to host in Staphylococcus aureus [115], and the ppdC gene involved in extracellular secretion machinery in Pseudomonas aeruginosa [116]. Additionally, we also observed many predicted interaction factors that are physically clustered together on the chromosome. For instance, our prediction list includes an 11-gene cluster for a general secretion system, and a 12-gene cluster that may be associated with type IV secretion complex formation. This result agrees with previous studies that many virulence properties of microbes are a collaborative effort of multiple genes and their physical clustering (and/or co-expression as operons) is under functional and evolutionary constraints $[117,118]$.

Interestingly, our predicted host-microbe interaction factor lists include at least 17 chemotaxis or motility associated proteins for each organism, including putative methyl-accepting chemotaxis receptors and one type IV pilus biogenesis protein involved in bacterial motility and adhesion to a solid surface [119]. Previous studies have indicated the chemotactic responses with specific cellular localization are critical for biofilm formation and interaction with hosts in a variety of pathogenic bacteria [120-124]. The hypergeometric distribution was used to assess the statistical significance of enrichment of a given functional group in the target list relative to the genome as a whole $[125,126]$. Interpro family annotations were uniformly assigned across both genomes and we conducted enrichment tests based on assignment to the Interpro chemotaxis family. The highly significant $\mathrm{p}$-values for both Dd3937 ( $\mathrm{p}=3.42 \mathrm{e}-11)$ and WPP14 $(\mathrm{p}=3.36 \mathrm{e}-12)$ strongly suggest methyl-accepting chemotaxis genes are highly enriched among the predicted host-microbe interaction factors.

Our learning strategy was explicitly designed to separate genes likely to be involved in host-microbe interaction from genes involved with core biological processes. The evidence above strongly suggests that the method is effective at recognizing host-microbe interaction factors, but it is important to keep in mind that it does not directly address the possibility that some genes associated with core 
biological processes may also contribute to interaction with hosts. Direct experimental testing of a relatively large number of genes from both the positive and negative classes is underway and will illuminate the power of this machine learning approach to guide discovery.

\section{Conclusion}

Although bacterial pathogen genome sequencing has become routine, the large number of unknown genes has been, and still is, a major obstacle to understanding the mechanisms of infection and adaptive evolution of microbial pathogens overall. We successfully employed supervised machine learning to identify candidate host interaction factors and we are able to predict host-microbe interaction factors from among genes of entirely unknown function, for two important agricultural pathogens Dickeya dadantii Dd3937 and Pectobacterium carotovorum WPP14, achieving promising results with a precision rate over $90 \%$ with a recall rate over $80 \%$. The predictions made in this study include many genes that have not previously been linked to host microbe interaction, a result not achievable with homology-based search strategies, providing an expanded list of appealing targets for further experimental validation. Our results indicate the learning schemes used in this study can recognize the complex patterns of hostmicrobe interaction factors and yield biologically meaningful results. Because of the powerful and intelligent models supervised machine learning schemes are capable of constructing, their future application to studying additional complex biological processes is likely to be a productive research approach.

\section{Availability of supporting data}

The data sets supporting the results of this article are available in the LabArchives repository, [https://mynotebook. labarchives.com/share/plantpath/MjAuOHwyNTc2OC8xN i9UcmVlTm9kZS8yNjQ4MTE0NTEOfDUyLjg=].

\section{Additional files}

Additional file 1: List of individual protein-coding genes from Dd3937 (a) and WPP14 (b) assigned host-microbe interaction GO annotations corresponding to Table 2 .

Additional file 2: (a) Strain information of all gamma-proteobacteria with complete or nearly complete genomes used in this study and their associations with various phenotypes of interest (as listed in Additional file 2: Table S1). (b) List of all non gamma-proteobacteria with complete or nearly complete genomes used in this study. (c) List of attribute categories used to generate summary taxonomic and phenotypic attributes based on sequence homology, the count of genomes in each category used as BLASTP search databases, and the number of proteins in Dickeya dadantii (Dd3937) and Pectobacterium carotovorum (WPP14) with hits to target organisms in those categories. (d) List of attributes used in the category of phenotypes of interest, including taxonomic groups and lifestyle statistics.

Additional file 3: Functional genomics data sets used in this study. (a) Data sets generated from microarray or proteomics studies. (b) Binding sites prediction of 32 putative transcriptional factors that may be related with host-microbe interactions.

Additional file 4: Description of selected supervised machine learning schemes.

Additional file 5: (a) List of selected attributes with importance measurement score and subsets of attributes defined to be used in data decay analysis for Dickeya dadantii 3937 and Pectobacterium carotovorum WPP14, (b) probability score plot used to define a compact set of attributes for both strains.

Additional file 6: (a) RP curve to compare strategies of data transformation for boosting classifier performance. (b) PR curve to compare classifier performance using five data sets partitioned according to GO terms for different aspects of host-microbe interaction (refer to Table 2).

Additional file 7: The list of predicted host-microbe interaction factors. (a) List of 211 genes predicted to be host-microbe interaction factors for Dickeya dadantii 3937, (b) list of 216 genes predicted to be host-microbe interaction factors for Pectobacterium carotovorum WPP14. (c) list of top 300 genes that could not be confidently classified as host-microbe interaction factors for both Dickeya dadantii 3937 and Pectobacterium carotovorum WPP14.

\section{Abbreviations}

RF: Random forest; SMO: Support Vector Machine using Sequential Minimal Optimization; poly: SMO using polynomial kernels; rbf: SMO using Radial Basis Function (RBF) kernels; ab: Adaptive boosting; j48: C4.5 algorithm in decision tree; mb: Multi-boosting; nb: Naive bayes; Ib: Logitboost; ds: Decision stump; BN: Bayesian network.

\section{Competing interest}

The authors declare that there are no competing interests.

\section{Authors' contributions}

BM, NTP, JDG designed the study. BM performed the analyses. AOC and NTP assigned the category of target class. BM, AOC, and NTP interpret the data. $B M, A O C$, JDG, and NTP wrote the manuscript. All authors read and approved the final manuscript.

\section{Acknowledgement}

We would like to thank Dr. Pablo Rodriquez-Palenzuela from Technical Univ. of Madrid for assisting with assignment of annotations and assembling the training set, Dr. C. David Page Jr. from UW-Madison for providing advice on machine learning analyses, and Andrew Tritt for help in setting up the transcriptional regulation factor prediction analysis. BM was supported by Morgridge Institute for Research (MIR) funds in the Computation \& Informatics in Biology \& Medicine (CIBM) Training Program.

\section{Author details}

'Genome Center of Wisconsin, University of Wisconsin-Madison, Madison, WI 53706, USA. ${ }^{2}$ Department of Plant Pathology, University of Wisconsin-Madison, Madison, WI 53706, USA. ${ }^{3}$ Department of Genetics, University of Wisconsin, Madison, WI 53706, USA. ${ }^{4}$ Current affiliation: Institute for Genome Sciences, University of Maryland School of Medicine, Baltimore, MD 21201, USA.

Received: 22 December 2013 Accepted: 9 June 2014 Published: 21 June 2014

\section{References}

1. Hayes WS, Borodovsky M: How to interpret an anonymous bacterial genome: machine learning approach to gene identification. Genome Res 1998, 8(11):1154-1171.

2. Hamady M, Betterton MD, Knight $R$ : Using the nucleotide substitution rate matrix to detect horizontal gene transfer. BMC Bioinform 2006, 7:476.

3. Lewis DP, Jebara T, Noble WS: Support vector machine learning from heterogeneous data: an empirical analysis using protein sequence and structure. Bioinformatics 2006, 22(22):2753-2760.

4. Matukumalli LK, Grefenstette JJ, Hyten DL, Choi IY, Cregan PB, Van Tassell CP: Application of machine learning in SNP discovery. BMC Bioinform 2006, 7:4. 
5. Brown MPS, Grundy WN, Lin D, Cristianini N, Sugnet CW, Furey TS, Ares M Haussler D: Knowledge-based analysis of microarray gene expression data by using support vector machines. Proc Natl Acad Sci U S A 2000, 97(1):262-267.

6. Hugouvieux-Cotte-Pattat N, Condemine G, Nasser W, Reverchon S: Regulation of pectinolysis in Erwinia chrysanthemi. Annu Rev Microbiol 1996, 50:213-257.

7. Glasner JD, Marquez-Villavicencio M, Kim HS, Jahn CE, Ma B, Biehl BS, Rissman Al, Mole B, Yi X, Yang CH, Dangl JL, Grant SR, Perna NT, Charkowski AO: Niche-specificity and the variable fraction of the Pectobacterium pan-genome. Mol Plant Microbe Interact 2008, 21(12):1549-1560.

8. Barras F, Vangijsegem F, Chatterjee AK: Extracellular Enzymes and Pathogenesis of Soft-Rot Erwinia. Annu Rev Phytopathol 1994, 32:201-234.

9. Lakso JU, Starr MP: Comparative injuriousness to plants of Erwinia spp. and other enterobacteria from plants and animals. J Appl Bact 1970, 33:692-707.

10. Gardan L, Gouy C, Christen R, Samson R: Elevation of three subspecies of Pectobacterium carotovorum to species level: Pectobacterium atrosepticum sp nov., Pectobacterium betavasculorum sp nov and Pectobacterium wasabiae sp nov. Int I Syst Evol Microbiol 2003, 53:381-391.

11. Ma B, Hibbing ME, Kim HS, Reedy RM, Yedidia I, Breuer J, Breuer J, Glasner JD, Perna NT, Kelman A, Charkowski AO: Host range and molecular Phylogenies of the soft rot enterobacterial genera Pectobacterium and dickeya. Phytopathology 2007, 97(9):1150-1163.

12. Ried JL, Collmer A: Comparison of pectic enzymes produced by Erwinia chrysanthemi, Erwinia carotovora subsp. carotovora, and Erwinia carotovora subsp. atroseptica. Appl Environ Microbiol 1986, 52(2):305-310.

13. Yang C-H, Gavilanes-Ruiz M, Okinaka Y, Vedel R, Berthuy I, Boccara M, Chen JWT, Perna NT, Keen NT: hrp genes of Erwinia chrysanthemi 3937 are important virulence factors. Mol Plant Microbe Interact 2002, 15(5):472-480.

14. Gloux K, Touze T, Pagot Y, Jouan B, Blanco C: Mutations of ousA alter the virulence of Erwinia chrysanthemi. Mol Plant Microbe Interact 2005 18(2):150-157.

15. Venkatesh B, Babujee L, Liu H, Hedley P, Fujikawa T, Birch P, Toth I, Tsuyumu S: The Erwinia chrysanthemi $3937 \mathrm{PhoQ}$ sensor kinase regulates several virulence determinants. J Bacterio/ 2006, 188(8):3088-3098.

16. Lopez-Solanilla E, Llama-Palacios A, Collmer A, Garcia-Olmedo F, Rodriguez-Palenzuela P: Relative effects on virulence of mutations in the sap, pel, and hrp loci of Erwinia chrysanthemi. Mol Plant Microbe Interact 2001, 14(3):386-393.

17. Rouanet C, Reverchon S, Rodionov DA, Nasser W: Definition of a consensus DNA-binding site for $\mathrm{PecS}$, a global regulator of virulence gene expression in Erwinia chrysanthemi and identification of new members of the PecS regulon. J Biol Chem 2004, 279(29):30158-30167.

18. Yap M-N, Yang CH, Barak JD, Jahn CE, Charkowski AO: The Erwinia chrysanthemi type III secretion system is required for multicellular behavior. J Bacteriol 2005, 187:639-648.

19. Herault E, Reverchon S, Nasser W: Role of the LysR-type transcriptional regulator PecT and DNA supercoiling in the thermoregulation of pel genes, the major virulence factors in Dickeya dadantii. Environ Microbiol 2013, 16:3. 734-745.

20. Bradbury JF: Erwinia. In Guide to plant pathogenic bacteria. Wallingford, UK: CAB International; 1986:61-79.

21. Knauss JF, Miller JW: Bacterial blight of Saintpaulia ionantha caused by Erwinia chrysanthemi. Phytopathol 1974, 64:1046-1047.

22. Yap MN, Barak JD, Charkowski AO: Genomic diversity of Erwinia carotovora subsp. carotovora and its correlation with virulence. Appl Environ Microbiol 2004, 70(5):3013-3023.

23. Perombelon MCM: Potato diseases caused by soft rot erwinias: an overview of pathogenesis. Plant Pathol 2002, 51(1):1-12.

24. Toth IK, Bell KS, Holeva MC, Birch PRJ: Soft rot erwiniae: from genes to genomes. Mol Plant Pathol 2003, 4(1):17-30.

25. Glasner JD, Liss P, Plunkett G III, Darling A, Prasad T, Rusch M, Byrnes A Gilson M, Biehl B, Blattner FR, Perna NT: ASAP, a systematic annotation package for community analysis of genomes. Nucleic Acids Res 2003, 31(1):147-151.

26. Glasner JD, Rusch M, Liss P, Plunkett G 3rd, Cabot EL, Darling A, Anderson BD, Infield-Harm P, Gilson MC, Perna NT: ASAP: a resource for annotating, curating, comparing, and disseminating genomic data. Nucleic Acids Res 2006, 34(Database issue):D41-D45

27. Hommais F, Oger-Desfeux C, Van Gijsegem F, Castang S, Ligori S, Expert D, Nasser W, Reverchon S: PecS Is a Global Regulator of the Symptomatic
Phase in the Phytopathogenic Bacterium Erwinia chrysanthemi 3937. J Bacteriol 2008, 190(22):7508-7522.

28. Ravirala RS, Barabote RD, Wheeler DM, Reverchon S, Tatum O, Malouf J, Liu H, Pritchard L, Hedley PE, Birch PR, Toth IK, Payton P, San Francisco MJ: Efflux pump gene expression in Erwinia chrysanthemi is induced by exposure to phenolic acids. Mol Plant Microbe Interact 2007, 20(3):313-320.

29. Mattinen L, Somervuo P, Nykyri J, Nissinen R, Kouvonen P, Corthals G, Auvinen P, Aittamaa M, Valkonen JP, Pirhonen M: Microarray profiling of host-extract-induced genes and characterization of the type $\mathrm{VI}$ secretion cluster in the potato pathogen Pectobacterium atrosepticum. Microbiology 2008, 154(Pt 8):2387-2396.

30. Rodionov DA, Gelfand MS, Hugouvieux-Cote-Pattat N: Comparative genomics of the KdgR regulon in Erwinia chrysanthemi 3937 and other gamma-proteobacteria. Microbiol 2004, 150:3571-3590.

31. Nasser W, Faelen M, Hugouvieux-Cotte-Pattat N, Reverchon S: Role of the nucleoid-associated protein $\mathrm{H}$-NS in the synthesis of virulence factors in the phytopathogenic bacterium Erwinia chrysanthemi. Mol Plant Microbe Interact 2001, 14(1):10-20.

32. Nasser W, Reverchon S: H-NS-dependent activation of pectate lyases synthesis in the phytopathogenic bacterium Erwinia chrysanthemi is mediated by the PecT repressor. Mol Microbio/ 2002, 43(3):733-748.

33. Rodionov DA, Mironov AA, Rakhmaninova AB, Gelfand MS: Transcriptional regulation of transport and utilization systems for hexuronides, hexuronates and hexonates in gamma purple bacteria. Mol Microbiol 2000, 38(4):673-683.

34. Zheng D, Constantinidou C, Hobman JL, Minchin SD: Identification of the CRP regulon using in vitro and in vivo transcriptional profiling. Nucleic Acids Res 2004, 32(19):5874-5893.

35. Li ZR, Lin HH, Han LY, Jiang L, Chen $X$, Chen YZ: PROFEAT: a web server for computing structural and physicochemical features of proteins and peptides from amino acid sequence. Nucleic Acids Res 2006, 34(Web Server issue):W32-W37.

36. Price MN, Huang KH, Alm EJ, Arkin AP: A novel method for accurate operon predictions in all sequenced prokaryotes. Nucleic Acids Res 2005, 33(3):880-892

37. Tatusov RL, Natale DA, Garkavtsev IV, Tatusova TA, Shankavaram UT, Rao BS, Kiryutin B, Galperin MY, Fedorova ND, Koonin EV: The COG database: new developments in phylogenetic classification of proteins from complete genomes. Nucleic Acids Res 2001, 29(1):22-28.

38. Gouy M, Gautier C: Codon usage in bacteria: correlation with gene expressivity. Nucleic Acids Res 1982, 10(22):7055-7074.

39. Bennetzen JL, Hall BD: Codon selection in yeast. J Biol Chem 1982, 257(6):3026-3031

40. Pellegrini M, Marcotte EM, Thompson MJ, Eisenberg D, Yeates TO: Assigning protein functions by comparative genome analysis: protein phylogenetic profiles. Proc Natl Acad Sci U S A 1999, 96(8):4285-4288.

41. Eisenberg D, Marcotte EM, Xenarios I, Yeates TO: Protein function in the post-genomic era. Nature 2000, 405(6788):823-826.

42. Yu NY, Wagner JR, Laird MR, Melli G, Rey S, Lo R, Dao P, Sahinalp SC, Ester M, Foster $\sqcup$, Brinkman FS: PSORTb 3.0: improved protein subcellular localization prediction with refined localization subcategories and predictive capabilities for all prokaryotes. Bioinformatics 2010, 26(13):1608-1615.

43. Attwood TK, Avison H, Beck ME, Bewley M, Bleasby AJ, Brewster F, Cooper P, Degtyarenko K, Geddes AJ, Flower DR, Kelly MP, Lott S, Measures KM, Parry-Smith DJ, Perkins DN, Scordis P, Scott D, Worledge C: The PRINTS database of protein fingerprints: A novel information resource for computational molecular biology. J Chem Inf Comput Sci 1997, 37(3):417-424.

44. Attwood TK, Blythe MJ, Flower DR, Gaulton A, Mabey JE, Maudling N, McGregor L, Mitchell AL, Moulton G, Paine K, Scordis P: PRINTS and PRINTS-S shed light on protein ancestry. Nucleic Acids Res 2002, 30(1):239-241.

45. Demerec M, Hartman PE: Complex loci in microorganisms. Annu Rev Microbiol 1959, 13:377-406

46. Rey S, Gardy JL, Brinkman FS: Assessing the precision of high-throughput computational and laboratory approaches for the genome-wide identification of protein subcellular localization in bacteria. BMC Genomics 2005, 6:162

47. Sharp PM, Li WH: The codon Adaptation Index-a measure of directional synonymous codon usage bias, and its potential applications. Nucleic Acids Res 1987, 15(3):1281-1295. 
48. Grote A, Hiller K, Scheer M, Munch R, Nortemann B, Hempel DC, Jahn D: JCat: a novel tool to adapt codon usage of a target gene to its potential expression host. Nucleic Acids Res 2005, 33(Web Server issue):W526-W531.

49. Overbeek R, Fonstein M, D'Souza M, Pusch GD, Maltsev N: The use of gene clusters to infer functional coupling. Proc Natl Acad Sci U S A 1999, 96(6):2896-2901.

50. Dandekar T, Snel B, Huynen M, Bork P: Conservation of gene order: a fingerprint of proteins that physically interact. Trends Biochem Sci 1998, 23(9):324-328.

51. Price $M N$, Huang $\mathrm{KH}$, Arkin AP, Alm EJ: Operon formation is driven by co-regulation and not by horizontal gene transfer. Genome Res 2005, 15(6):809-819.

52. Tatusov RL, Koonin EV, Lipman DJ: A genomic perspective on protein families. Science 1997, 278:631-637

53. Langille MG, Hsiao WW, Brinkman FS: Evaluation of genomic island predictors using a comparative genomics approach. BMC Bioinform 2008, 9:329

54. Langille MG, Brinkman FS: IslandViewer: an integrated interface for computational identification and visualization of genomic islands. Bioinformatics 2009, 25(5):664-665.

55. Rao HB, Zhu F, Yang GB, Li ZR, Chen YZ: Update of PROFEAT: a web server for computing structural and physicochemical features of proteins and peptides from amino acid sequence. Nucleic Acids Res 2011, 39(Web Server issue):W385-W390

56. Hall M, Frank E, Holmes G, Pfahringer B, Reutemann P, Witten $I H$ : The WEKA Data Mining Software: An Update. SIGKDD Explor 2009, 11(1):10-18.

57. Witten $I H$, Eibe F, Hall MA: Data Mining: Practical machine learning tools and techniques. 3rd edition. Burlington, MA, USA: Elsevier; 2005.

58. Quinlan R: C4.5: Programs for Machine Learning. San Mateo, CA: Morgan Kaufmann Publishers; 1993

59. Hastie T, Tibshirani R: Classification by Pairwise Coupling. Annu Stat 1998 26(2):451-471

60. Keerthi SS, Shevade SK, Bhattacharyya C, Murthy KRK: Improvements to Platt's SMO Algorithm for SVM Classifier Design. Neural Comput 2001, 13(3):637-649.

61. Platt J: Machines using Sequential Minimal Optimization. In Advances in Kernel Methods - Support Vector Learning. Edited by Schoelkopf B, Burges C, Smola A. Cambridge, MA, USA: The MIT Press; 1998.

62. Cooper GF, Herskovits E: A Bayesian method for constructing Bayesian belief networks from databases. In UAl'91 Proceedings of the Seventh conference on Uncertainty in Artificial Intelligence. San francisco, CA, USA: Morgan Kaufmann Publishers Inc; 1991:86-94.

63. Cooper GF, Herskovits E: A Bayesian method for the induction of probabilistic networks from data. Mach Learn 1992, 9(4):309-347.

64. George HJ, Langley P: Estimating Continuous Distributions in Bayesian Classifiers, San Mateo. In UAl'95 Eleventh Conference on Uncertainty in Artificial Intelligence; 1995:338-345.

65. Aha D, Kibler D: Instance-based learning algorithms. Mach Learn 1991, 6:37-66

66. Cohen WW: Fast Effective Rule Induction. In Twelfth International Conference on Machine Learning; 1995:115-123.

67. Friedman J, Hastie T, Tibshirani R: Additive Logistic Regression: a Statistical View of Boosting. Annu Stat 2000, 28(2):337-407.

68. Breiman L: Bagging predictors. Mach Learn 1996, 24(2):123-140.

69. Webb Gl: MultiBoosting: a technique for combining boosting and wagging. Mach Learn 2000, 40(2):159-196.

70. Freund Y, Schapire RE: Experiments with a new boosting algorithm. In Thirteenth International Conference on Machine Learning. San Francisco; 1996:148-156.

71. Kittler J, Hatef M, Duin RPW, Matas J: On combining classifiers. IEEE Trans Pattern Anal Mach Intell 1998, 20(3):226-239.

72. Kuncheva LK: Combining Pattern Classifiers: Methods and Algorithms. Loboken, NJ: Wiley-Interscience; 2004.

73. Wolpert DH: Stacked generalization. Neural Netw 1992, 5:241-259

74. Kohavi R: A study of cross-validation and bootstrap for accuracy estimation and model selection. In Proceedings of the Fourteenth International Joint Conference on Artificial Intelligence 1995. San Mateo: Morgan Kaufmann; 1995:1137-1143

75. Devijver PA, Kittler J: Pattern Recognition: A Statistical Approach. London: Prentice-Hall; 1982

76. Peterson LE, Coleman MA: Machine learning-based receiver operating characteristic (ROC) curves for crisp and fuzzy classification of DNA microarrays in cancer research. International Journal of Approximate Reasoning 2008, 47(1):17-36.

77. Jolliffe IT: Principal Component Analysis, Volume 487. 2nd edition. NY: Springer; 2002.

78. Kononenko I: On Biases in Estimating Multi-Valued Attributes. In 14th International Joint Conference on Artificial Intelligence; 1995:1034-1040.

79. Fayyad U, Irani K: Multi-interval discretization of continuousvalued attributes for classification learning. In Thirteenth International Joint Conference on Articial Intelligence: 1993; 1993:1022-1027.

80. Breiman L: Random Forests. Mach Learn 2001, 45(1):5-32.

81. Breiman and Cutler's Random Forests for Classification and Regression. [http://CRAN.R-project.org/package=randomForest]

82. Livingston F: Implementation of Breimans Random Forest Machine Learning Algorithm, ECE591Q Machine Learning Journal Paper; 2005.

83. Provost F, Fawcett $T$, Kohavi R: The case against accuracy estimation for comparing induction algorithms. In Proceeding of the 15th International Conference on Machine Learning. San Francisco, CA: Morgan Kaufmann; 1998:445-453.

84. Davis J: The relationship between precision-recall and ROC curves. In Proceedings of the 23th International Conference on Machine Learning: 2006; Pittsburgh, PA; 2006.

85. Kalia A, Bessen DE: Natural selection and evolution of streptococcal virulence genes involved in tissue-specific adaptations. J Bacterio/ 2004 186(1):110-121.

86. Wiles TJ, Norton JP, Smith SN, Lewis AJ, Mobley HL, Casjens SR, Mulvey MA: A phyletically rare gene promotes the niche-specific fitness of an E. coli pathogen during bacteremia. PLoS Pathog 2013 9(2):e1003175.

87. Kohavi R, John GH: Wrappers for Feature Subset Selection. Artif Intell 1997, 97(1-2):273-324

88. Blum A, Langley P: Selection of relevant features and examples in machine learning. Artif Intell 1997, 97:245-271.

89. Hall MA: Correlation-based Feature Subset Selection for Machine Learning New Zealand: Hamilton; 1998

90. Guyon I, Weston J, Barnhill S, Vapnik V: Gene selection for cancer classification using support vector machines. Mach Learn 2002, 46:389-422

91. Lustgarten JL, Gopalakrishnan V, Grover H, Visweswaran S: Improving Classification Performance with Discretization on Biomedical Datasets. In AMIA Annu Symp Proc: 2008: 2008:445-449.

92. Liu H, Setiono R: Feature selection via discretization. Knowl Data Eng 1997, 9(4):642-645.

93. Landini P, Zehnder AJ: The global regulatory hns gene negatively affects adhesion to solid surfaces by anaerobically grown Escherichia coli by modulating expression of flagellar genes and lipopolysaccharide production. J Bacterio/ 2002, 184(6):1522-1529.

94. Harrison JA, Pickard D, Higgins CF, Khan A, Chatfield SN, Ali T, Dorman CJ, Hormaeche CE, Dougan G: Role of hns in the virulence phenotype of pathogenic salmonellae. Mol Microbiol 1994, 13(1):133-140.

95. Tetko IV, Livingstone DJ, Luik Al: Neural-Network Studies 1. Comparison of Overfitting and Overtraining. J Chem Inf Comput SC 1995, 35(5):826-833.

96. Sima C, Attoor S, Brag-Neto U, Lowey J, Suh E, Dougherty ER: Impact of error estimation on feature selection. Pattern Recogn 2005, 38(12):2472-2482.

97. Caruana R, Niculescu-Mizil A: An empirical comparison of supervised learning algorithms. In Proceedings of the 23rd international conference on Machine learning 2006; 2006.

98. Freund $Y$ : Boosting a weak learning algorithm by majority. Inform Comput 1995, 121(2):256-285.

99. Legault BA, Lopez-Lopez A, Alba-Casado JC, Doolittle WF, Bolhuis H, Rodriguez-Valera F, Papke RT: Environmental genomics of "Haloquadratum walsbyi" in a saltern crystallizer indicates a large pool of accessory genes in an otherwise coherent species. BMC Genomics 2006, 7:171.

100. Gill SR, Pop M, Deboy RT, Eckburg PB, Turnbaugh PJ, Samuel BS, Gordon الر, Relman DA, Fraser-Liggett CM, Nelson KE: Metagenomic analysis of the human distal gut microbiome. Science 2006, 312(5778):1355-1359.

101. Venter JC, Remington K, Heidelberg JF, Halpern AL, Rusch D, Eisen JA, Wu D, Paulsen I, Nelson KE, Nelson W, Fouts DE, Levy S, Knap AH, Lomas MW, Nealson K, White O, Peterson J, Hoffman J, Parsons R, Baden-Tillson H, Pfannkoch C, Rogers YH, Smith HO: Environmental genome shotgun sequencing of the Sargasso Sea. Science 2004, 304(5667):66-74. 
102. Edwards RA, Rodriguez-Brito B, Wegley L, Haynes M, Breitbart M, Peterson DM, Saar MO, Alexander S, Alexander EC Jr, Rohwer F: Using pyrosequencing to shed light on deep mine microbial ecology. BMC Genomics 2006, 7:57.

103. Galperin MY, Koonin EV: 'Conserved hypothetical' proteins: prioritization of targets for experimental study. Nucleic Acids Res 2004 32(18):5452-5463.

104. Braudo EE, Plashchina IG, Kobak W, Golovnya RV, Zhuravleva IL, Krikunova $\mathrm{Nl}$ : Interactions of flavor compounds with pectic substances. Food Nahrung 2000, 44(3):173-177.

105. Delangle A, Prouvost AF, Cogez V, Bohin JP, Lacroix JM, Cotte-Pattat NH: Characterization of the Erwinia chrysanthemi gan locus, involved in galactan catabolism. J Bacterio/ 2007, 189(19):7053-7061.

106. Mattinen L, Tshuikina M, Mae A, Pirhonen M: Identification and characterization of Nip, necrosis-inducing virulence protein of Erwinia carotovora subsp carotovora. Mol Plant Microbe Interact 2004, 17(12):1366-1375.

107. Fischer E, Gunter K, Braun V: Involvement of Exbb and Tonb in Transport across the Outer-Membrane of Escherichia-Coli - Phenotypic Complementation of Exb Mutants by Overexpressed Tonb and Physical Stabilization of Tonb by Exbb. J Bacterio/ 1989, 171(9):5127-5134.

108. Wiggerich $H G$, Puhler $A$ : The exbD2 gene as well as the iron-uptake genes ton $\mathrm{B}$, exbB and exbD1 of Xanthomonas campestris pv. campestris are essential for the induction of a hypersensitive response on pepper (Capsicum annuum). Microbiol-Sgm 2000, 146:1053-1060.

109. Bitter W, Tommassen J, Weisbeek PJ: Identification and Characterization of the Exbb, Exbd and Tonb Genes of Pseudomonas-Putida Wcs358 - Their Involvement in Ferric-Pseudobactin Transport. Mol Microbiol 1993 7(1):117-130

110. Watson RJ, Joyce SA, Spencer GV, Clarke DJ: The exbD gene of Photorhabdus temperata is required for full virulence in insects and symbiosis with the nematode Heterorhabditis. Mol Microbio/ 2005, 56(3):763-773.

111. Bjarnason J, Southward CM, Surette MG: Genomic profiling of iron-responsive genes in Salmonella enterica serovar typhimurium by high-throughput screening of a random promoter library. J Bacterio/ 2003, 185(16):4973-4982.

112. Said-Salim B, Dunman PM, McAleese FM, Macapagal D, Murphy E, McNamara PJ, Arvidson S, Foster TJ, Projan SJ, Kreiswirth BN: Global regulation of Staphylococcus aureus genes by rot. J Bacteriol 2003, 185(2):610-619.

113. De Maayer P, Chan WY, Venter SN, Toth IK, Birch PR, Joubert F, Coutinho TA: Genome sequence of Pantoea ananatis LMG20103, the causative agent of Eucalyptus blight and dieback. J Bacterio/ 2010, 192(11):2936-2937.

114. Cao H, Krishnan G, Goumnerov B, Tsongalis J, Tompkins R, Rahme LG: A quorum sensing-associated virulence gene of Pseudomonas aeruginosa encodes a LysR-like transcription regulator with a unique self-regulatory mechanism. Proc Natl Acad Sci U S A 2001, 98(25):14613-14618.

115. Marraffini LA, Dedent AC, Schneewind O: Sortases and the art of anchoring proteins to the envelopes of gram-positive bacteria. Microbio/ Mol Biol Rev 2006, 70(1):192-221.

116. Strom MS, Nunn DN, Lory S: Posttranslational processing of type IV prepilin and homologs by PilD of Pseudomonas aeruginosa. Methods Enzymol 1994, 235:527-540.

117. Korbel JO, Jensen LJ, von Mering C, Bork P: Analysis of genomic context: prediction of functional associations from conserved bidirectionally transcribed gene pairs. Nat Biotechnol 2004, 22(7):911-917.

118. Davila Lopez M, Martinez Guerra JJ, Samuelsson T: Analysis of gene order conservation in eukaryotes identifies transcriptionally and functionally linked genes. PloS One 2010, 5(5):e10654.

119. Mattick JS: Type IV pili and twitching motility. Annu Rev Microbiol 2002, 56:289-314.

120. Lybarger SR, Maddock JR: Polarity in action: asymmetric protein localization in bacteria. J Bacteriol 2001, 183(11):3261-3267.

121. Bardy SL, Maddock JR: Polar localization of a soluble methyl-accepting protein of Pseudomonas aeruginosa. J Bacteriol 2005, 187(22):7840-7844

122. Yao J, Allen C: The plant pathogen Ralstonia solanacearum needs aerotaxis for normal biofilm formation and interactions with its tomato host. J Bacterio/ 2007, 189(17):6415-6424.

123. Hagman KE, Porcella SF, Popova TG, Norgard MV: Evidence for a methyl-accepting chemotaxis protein gene $(\mathrm{mcp} 1)$ that encodes a putative sensory transducer in virulent Treponema pallidum. Infect Immun 1997, 65(5):1701-1709.
124. Collmer A, Keen NT: The role of pectic enzymes in plant pathogenesis. Annu Rev Phytopathol 1986, 24:383-409.

125. Rivals I, Personnaz L, Taing L, Potier MC: Enrichment or depletion of a GO category within a class of genes: which test? Bioinformatics 2007, 23(4):401-407.

126. Draghici S, Khatri P, Martins RP, Ostermeier GC, Krawetz SA: Global functional profiling of gene expression. Genomics 2003, 81(2):98-104.

doi:10.1186/1471-2164-15-508

Cite this article as: Ma et al:: Identification of host-microbe interaction factors in the genomes of soft rot-associated pathogens Dickeya dadantii 3937 and Pectobacterium carotovorum WPP14 with supervised machine learning. BMC Genomics 2014 15:508.

\section{Submit your next manuscript to BioMed Central and take full advantage of:}

- Convenient online submission

- Thorough peer review

- No space constraints or color figure charges

- Immediate publication on acceptance

- Inclusion in PubMed, CAS, Scopus and Google Scholar

- Research which is freely available for redistribution 